\title{
Eficacia de un programa de preparación psicológica en jugadores jóvenes de fútbol
}

\author{
Isabel María Moreno-Fernández', Verónica Gómez-Espejo², Belén Olmedilla-Caballero², Luis Miguel Ramos-Pastra- \\ na $^{3}$, Enrique Ortega-Toro ${ }^{2}$ y Aurelio Olmedilla-Zafra ${ }^{2}$ \\ ${ }^{1}$ Universidad Autónoma de Barcelona, España ${ }^{2}$ Universidad de Murcia, España ${ }^{3}$ Universidad Autónoma de Madrid, España
}

RESUMEN: El objetivo del presente estudio fue determinar la eficacia de un programa de intervención psicológica (atención-concentración, motivación y nivel de activación) en 19 jugadores de fútbol 11, con una media de edad de $16.3(D T=.99)$. Se realizó una evaluación inicial y final mediante el Cuestionario de Características Psicológicas relacionadas con el Rendimiento Deportivo (CPRD) para valorar la eficacia de dicho programa. Los resultados indican diferencias estadísticamente significativas entre el pre y pos intervención, en el cual los jugadores mejoran en los factores del CPRD: "Influencia de Evaluación del Rendimiento", "Habilidad Mental" y "Control de Estrés". Se concluye que, el presente trabajo aporta evidencia sobre la eficacia de la intervención psicológica en el fútbol base, tal y como muestran los resultados.

PALABRAS CLAVES: Entrenamiento psicológico, fútbol, rendimiento, CPRD.

\section{Effectiveness of a psychological preparation programme in young football players}

ABSTRACT: The objective of this study was to assess the effectiveness of a psychological intervention programme (attentionconcentration, motivation, and activation level) in 19 football players with a mean age of $16.3(S D=.99)$. Effectiveness was assessed at baseline and at the end of the programme using the Psychological Characteristics related to Sports Performance (CPRD) questionnaire. There were statistically significant differences in the results found after the intervention, with players improving in the CPRD factors "Influence of Performance Evaluation", "Mental Ability", and "Stress Control". In conclusion, this paper provides evidence of the effectiveness of psychological interventions in grassroots football, as the results show.

KEYWORDS: Psychological training, football, performance, CPRD.

\section{Eficácia de um programa de preparação psicológica em jovens jogadores de futebol}

RESUMO: O objetivo do presente estudo foi determinar a eficácia de um programa de intervenção psicológica (atenção-concentração, motivação e nível de ativação) em 19 jogadores de futebol: 11, com idade média de 16,3 anos (DT=0,99). Foi realizada uma avaliação inicial e final através do Questionário de Características Psicológicas relacionadas com o Desempenho Desportivo (CPDD) para avaliar a eficácia desse programa. Os resultados indicam diferenças estatisticamente significativas entre a pré-intervenção e a pós-intervenção, em que os jogadores apresentam melhorias nos fatores das CPDD: "Influência da avaliação de desempenho", "Capacidade mental" e "Controlo do stress". Conclui-se que o presente trabalho fornece provas sobre a eficácia da intervenção psicológica no futebol, tal como mostram os resultados.

PALAVRAS-CHAVE: treino psicológico, futebol, desempenho, CPDD.

Artículo recibido: 07/09/2019 | Artículo aceptado: 21/10/2019

Financiación: Convenio entre la Universidad de Murcia y la Federación de Fútbol de la Región de Murcia (FFRM+UMU, N0: $040092321 \mathrm{~B} 6450214704)$ y al Contrato-Programa con el Real Murcia C.F. (RM-UMU-8141-19256).

Isabel María Moreno-Fernández es psicóloga en la Universidad Autónoma de Barcelona. Verónica Gómez-Espejo es psicóloga en la Universidad de Murcia. Belén Olmedilla-Caballero es estudiante de Psicología. Universidad de Murcia. Luis Miguel Ramos Pastrana es psicólogo en la Universidad Autónoma de Madrid. Enrique Ortega Toro es licenciado en Ciencias del Deporte por la Universidad de Granada. Aurelio Olmedilla Zafra es psicólogo Universidad de Murcia. La correspondencia sobre este artículo debe enviarse a la primera autora a Carrer de Provença, 54, $3^{\circ}$ 7ra, 08029, Barcelona (España). Mail: 
Es un hecho la gran importancia que tiene hoy en día la intervención psicológica en el deporte, además de por un mayor bienestar psicológico y la optimización del rendimiento deportivo, también por cómo el entrenamiento y/o enseñanza de estrategias psicológicas posibilitan la mejora de habilidades psicológicas tales como la concentración, la motivación, el nivel de activación, etc., que son tan importantes en la práctica deportiva (McCormick, Meijen y Marcora, 2018; Olmedilla, Bazaco, Ortega y Boladeras, 2011; Olmedilla y Domínguez-Igual, 2016;). En deportes con unas exigencias cambiantes, donde hay que realizar una toma de decisiones complejas continuamente (como los colectivos), las habilidades y destrezas cognitivas tienen tanta o incluso más relevancia que la ejecución técnica o táctica (Craig, 2011; Escolano, Herrero y Echeverría, 2014; Larkin, Mesagno, Berry, Spittle y Harvey, 2018; Olmedilla et al., 2018).

Las habilidades psicológicas en sí mismas no producen un rendimiento del deportista por encima de sus posibilidades, sino que pueden ayudar a conseguir, junto con el entrenamiento físico, táctico y técnico, que este deportista alcance un nivel de rendimiento lo más cercano posible a su potencial máximo (Olmedilla, Ortega, Boladeras, González y Serpa, 2015). El trabajo psicológico, que normalmente ha estado enmarcado dentro de la terapia cognitivo-conductual (Lindern, 2016; Mostaan, Sanatkaran y Bahari, 2015), se ha ido implantando en el plan de entrenamientos de muchos equipos deportivos, obteniendo muy buenos resultados en múltiples intervenciones, como en enseñanza de estrategias para la mejora de habilidades mentales (Lorenzo, Gómez, Pujals y Lorenzo, 2012) y en control emocional (Olmedilla, Ortega, Andreu y Ortín, 2010), entre otros. Tal y como señalan Nieto y Olmedilla (2001, p.128), "todos los aspectos generales del entrenamiento deportivo pueden verse beneficiados por la intervención psicológica, optimizando así la metodología de trabajo propia de entrenadores y deportistas". En todas las disciplinas deportivas está cobrando importancia la necesidad de llevar a cabo intervenciones psicológicas para estimular el bagaje psicológico de los deportistas, sobre todo en los deportes colectivos, en los que son muy importantes aspectos como la cohesión de equipo para el resultado final; lo que ha dado lugar a que, en estos últimos años, el entrenamiento psicológico se haya ido incluyendo cada vez más en la metodología de trabajo de equipos y deportistas, como puede verse en numerosos trabajos (Abenza et al., 2014; Carmona, Guzmán y Olmedilla, 2015; Olmedilla et al., 2015).

Tal y como señalan González y Garcés de los Fayos (2009, p. 91), el entrenamiento psicológico "es la práctica habitual de determinadas habilidades psicológicas como estrategias de afrontamiento en situaciones deportivas, tanto de entrenamiento como de competición". El objetivo central de este entrenamiento mental es "favorecer, eliminar o fortalecer algunas de las relaciones que se dan en la competición, con la finalidad de aumentar o mantener el propio rendimiento deportivo" (Mora, Zarco y Blanca, 2001, p.50). Algunos trabajos han relacionado directamente las características psicológicas de las deportistas y su percepción del rendimiento (Olmedilla, Ruiz-Barquín, Ponseti, Robles-Palazón y García-Mas, 2019). Sin embargo, es importante mencionar, siguiendo a Eraña (2004), que el entrenamiento psicológico no sólo va dirigido a un mejor o mayor rendimiento en los partidos, sino también a cómo entrenar mejor y a cómo planificar de manera correcta tanto partidos como entrenamientos. Éste provee al deportista de una serie de herramientas para que gestione adecuadamente sus recursos psicológicos de forma autónoma y ajustada a las exigencias de su deporte y de las diversas situaciones que en él se produzcan, para así controlar en todo lo posible su actuación, maximizar el rendimiento y el grado de control sobre su actuación y ampliar las opciones de éxito (ya sea en competición o en entrenamiento) (Godoy-Izquierdo, Vélez y Pradas, 2009; González y Garcés de los Fayos, 2009; Olmedilla et al., 2011).

Además, no es lo mismo trabajar con un deportista individual que con un deportista que forma parte de un equipo o con un equipo en sí: trabajar con deportistas de manera individual posibilita un feedback más fiable y directo de la eficacia del programa, que cuando se trabaja con equipos o grupos deportivos. Con estos últimos no se tiene una visión tan clara del funcionamiento y eficacia del entrenamiento psicológico, puesto que aparecen otras variables como las relaciones intragrupales, la cohesión, los resultados grupales, etc., que pueden perturbar esta visión (Olmedilla et al., 2010).

En línea con lo anterior, el entrenamiento psicológico no sólo es importante en el deporte profesional o de élite, sino también en el deporte base; ya que si, por ejemplo, un jugador no está motivado para hacer natación, no se adherirá, o si las competiciones para una gimnasta son muy estresantes, aunque sea muy buena en su disciplina, no llegará al nivel de rendimiento al que aspira (Navarrón, Godoy-Izquierdo, Vélez, Ramírez-Molina y Jiménez-Torres, 2017). En la actualidad, el fútbol base está teniendo una gran repercusión dentro de la Psicología del Deporte aplicada, puesto que además de promocionar y mejorar la salud física, también es muy importante la gran influencia que tiene en el proceso de socialización de los deportistas (Gómez-Espejo, Aroca, Robles-Palazón y Olmedilla, 2017). Torregrosa y Cruz (2006) hacen mención del llamado "triángulo deportivo", conformado por deportista-entrenador-familia pero además, y tal como señalan Gómez-Espejo et al. (2017), también hay que tener en cuenta al grupo de iguales, a la organización deportiva, a 
los árbitros, etc. en la calidad de la experiencia deportiva de éstos deportistas.

Numerosos estudios evidencian la importancia y eficacia del entrenamiento psicológico en fútbol base. Por ejemplo, Carmona et al. (2015) realizaron una intervención basada en el establecimiento de objetivos y el moldeamiento del pase durante cuatro semanas con 13 jugadores de fútbol juvenil, constatando la efectividad de ésta, observando un aumento de la percepción de competencia específica del pase (pudiendo influir directamente en la mejora de variables como motivación, autoconfianza y control de la ansiedad); además, se constataron mejoras en cuanto al rendimiento del pase en la competición. Por otro lado, Olmedilla et al. (2010) llevaron a cabo un entrenamiento psicológico con 22 jugadores jóvenes de fútbol, obteniendo mejoras en las variables control de estrés, influencia de la evaluación del rendimiento y habilidad mental, aunque no se obtuvo significación estadística.

Por tanto, el objetivo de este estudio fue llevar a cabo un programa de intervención psicológica en dos equipos de fútbol 11 masculino y comprobar su eficacia respecto a aquellas habilidades psicológicas que median y/o influyen en el rendimiento deportivo. Se plantea la siguiente hipótesis: Los jugadores mejorarán sus habilidades psicológicas y la gestión y el control del estrés después del programa de intervención psicológica.

\section{Método}

Para este estudio se utilizó un diseño cuasi-experimental de intervención con pre-test-post-test.

\section{Participantes}

La muestra de este estudio estuvo compuesta por 19 jugadores de fútbol 11, pertenecientes a un equipo de la cate- goría Cadete $(n=8)$ y otro Juvenil $(n=11)$, con una edad media de 16.3 años $(D T=.99)$. La experiencia como jugadores federados es de 10.89 años $(D T=1.56)$.

La intervención se llevó a cabo por una psicóloga con una experiencia de 3 años como alumna interna en Psicología del Deporte, habiendo realizado prácticas en el Real Murcia Sociedad Anónima Deportiva y en el Centro de Alto Rendimiento de San Javier.

\section{Instrumentos}

Para evaluar las variables psicológicas se ha utilizado el cuestionario de Características Psicológicas relacionadas con el Rendimiento Deportivo (CPRD) de Gimeno, Buceta y Pérez-Llantada (2001). Está formado por 55 ítems agrupados en 5 escalas: Control del Estrés (CE), Influencia de la Evaluación del Rendimiento (IER), Motivación (M), Habilidad Mental (HM) y Cohesión de Equipo (CE). Las respuestas están formuladas en una escala tipo Likert de 5 puntos, donde 0 es "totalmente en desacuerdo" y 4 es "totalmente de acuerdo. El alfa de Cronbach es de .85 para la escala total (Gimeno et al., 2001). Procedimiento

El programa de intervención se basó en la Terapia Cognitivo-Conductual (McArdle y Moore, 2012). Se realizó en 8 sesiones grupales por equipos, de 30-40 minutos cada una. En la Tabla 1 se muestra el cronograma y el contenido de las sesiones que se llevaron a cabo a los jugadores.

Antes de comenzar, hubo una breve reunión previa con ambos entrenadores (por separado) donde se les planteó tanto la propuesta de intervención psicológica como los objetivos perseguidos.

Todos los jugadores fueron informados del objetivo del estudio y de la confidencialidad tanto de sus respuestas como de los datos obtenidos posteriormente. Se obtuvo el consentimiento informado de todos los padres/tutores legales de los jugadores ya que éstos, en su mayoría, eran me-

Tabla 1. Cronograma y contenidos generales del programa

\begin{tabular}{lll}
\hline Febrero & Sesión 1 & Evaluación Inicial. \\
\hline Febrero & Sesión 2 & Motivación (I). \\
\hline Marzo & Sesión 3 & Motivación (II). Establecimiento objetivos. \\
\hline Marzo & Sesión 4 & Atención-Concentración (I). \\
\hline Marzo & Sesión 5 & Atención-Concentración (II). Visualización. \\
\hline Abril & Sesión 6 & Nivel de Activación (I). \\
\hline Abril & Sesión 7 & Nivel de Activación (II). Relajación. \\
\hline Abril & Sesión 8 & Evaluación Final. \\
\hline
\end{tabular}


nores de edad. Los 2 jugadores que sí eran mayores de edad dieron también por escrito su consentimiento.

En la Sesión 1 se explicó a los deportistas en qué consistía la intervención, qué se iba a trabajar y qué se esperaba de ellos. Además, se les explicaron conceptos como qué es la Psicología del Deporte, a qué se dedica el psicólogo del deporte y demás nociones básicas (ver Cantón, 2016), y posteriormente, hubo un turno de preguntas para que comentaran cualquier cosa que quisieran.

Las sesiones 2 y 3 versaron sobre la motivación, explicando en primer lugar los conocimientos clave como qué es, qué tipos hay y cómo se puede mantener o incluso aumentar (ver Clancy, Herring, MacIntyre, y Campbell, 2016). En la sesión 3, se explicaron también las nociones básicas sobre el establecimiento de objetivos (Carmona, Guzmán y Olmedilla, 2015) y se repartió a cada jugador dos autorregistros, uno sobre objetivos para el próximo partido y otro sobre una planificación de objetivos para lo que quedaba de temporada, con la intención de que lo cumplimentaran para corregir cómo los habían expresado, si eran medibles y factibles, etc.

Los objetivos de la temporada debían tenerlos siempre presentes de cara al resto de la misma, y antes de cada partido debían establecer dos objetivos a cumplir en él. El formato del autorregistro de objetivos para el partido era muy sencillo: debían plantear dos objetivos -importante que fueran objetivos de rendimiento y no de resultado- antes del partido y, al finalizar éste, debían describir si habían conseguido cumplirlo o no y describir los factores que habían ayudado o limitado la consecución del objetivo. Un ejemplo de objetivos que propusieron los jugadores fue: "correr al primer palo cuando Ángel saque el córner" (jugada ensayada que habían practicado en los entrenamientos). En cuanto al autorregistro de objetivos a largo plazo, debían proponer objetivos que debían cumplirse al final de la temporada, y añadir objetivos a corto y medio plazo que ayudaran en la consecución de estos objetivos finales. Un ejemplo de objetivo a largo plazo que propusieron fue "disminuir el número de goles en contra en partidos en los que juguemos como locales".

Las sesiones 4 y 5 estuvieron dirigidas al trabajo de la atención-concentración: qué es la atención y qué es la concentración, qué tipos de atención hay, cómo puede fluctuar la atención en el entrenamiento y en la competición, etc. (Morilla, 2006). En la sesión 5, se trabajó la técnica de visualización para la mejora de esta atención-concentración, explicando sus beneficios y cómo llevarla a cabo de una manera exitosa (González, 2006).

Las sesiones 6 y 7 versaron sobre el nivel de activación, explicando su concepto, la activación positiva y negativa y cómo influye en la competición y en el entrenamiento (López y Lozano, 2006). Además, en la sesión 7 se trabajó la técnica de Relajación Progresiva de Jacobson, enfocada a disminuir el nivel de activación, cómo practicarla y qué beneficios reporta al deportista (Díaz, Villalobos y Ruíz, 2012).

Por último, la sesión 8 se estableció como cierre, por lo que en una primera parte de la sesión se hizo un breve resumen de todo lo aprendido y una ronda de preguntas por si había dudas o algo que quisieran decir, y se les volvió a administrar el CPRD.

\section{Análisis de datos}

Se realizó un análisis estadístico descriptivo y la prueba t de student para muestras apareadas, utilizando un nivel de significación de $p<.05$.

Tabla 2. Puntuaciones media y diferencias ( $t$ de student) de las puntuaciones obtenidas en cada factor del CPRD $(n=19)$

\begin{tabular}{cccccccc}
\hline & Tiempo 1 & \multicolumn{7}{c}{ Tiempo 2} \\
& Media & DT & Media & DT & $t$ & $\begin{array}{c}\text { Sig. } \\
\text { (bilateral) }\end{array}$ & $d$ \\
\hline CE & 57.58 & 11.89 & 60.26 & 11.05 & -1.83 & .08 & .42 \\
\hline IER & 28.05 & 6.78 & 30.68 & 6.76 & -2.36 & .03 & .54 \\
\hline MO & 25.32 & 3.62 & 25.00 & 4.35 & -.45 & .65 & .10 \\
\hline HM & 22.05 & 3.19 & 24.32 & 2.52 & -2.35 & .03 & .54 \\
\hline CH & 18.84 & 2.39 & 18.05 & 3.01 & .94 & .36 & .15 \\
\hline
\end{tabular}

CE = Control de estrés; IER = Influencia de la Evaluación del Rendimiento; MO = Motivación; HM = Habilidad Mental; CH = Cohesión de Equipo. 


\section{Resultados}

En la Tabla 2, se presentan las medias de las puntuaciones obtenidas en el pretest y en el postest en cada uno de los factores. Al analizar las puntuaciones obtenidas y tal como se muestra en la Tabla 3, se puede observar que existen diferencias estadísticamente significativas en tres de los cinco factores, IER $(t(18)=-1.83 ; p=.03), \operatorname{HM}(t(18)=-2.35 ; p=.03)$ y $\operatorname{CE}(t(18)=-1.83 ; p=.08)$.

\section{Discusión}

El rendimiento deportivo está determinado por factores técnicos, tácticos, físicos y psicológicos. El entrenamiento psicológico es muy importante para la mejora y/o mantenimiento del rendimiento deportivo, produciendo resultados muy positivos en variables como la motivación, concentración, cohesión de equipo, etc. con técnicas como la visualización, el establecimiento de objetivos, relajación a través de la respiración, entre otros (Aoyagi, Poczwardowski, Statler, Shapiro y Cohen, 2017; Brown y Fletcher, 2017; McCormick et al., 2017). El objetivo de este estudio fue determinar la eficacia de un programa de intervención psicológica con jóvenes jugadores de fútbol.

Al analizar los datos de la muestra, los resultados indican que se encontraron diferencias estadísticamente significativas en tres factores, concretamente en IER, HM y CE. Partiendo de los criterios de Cohen (1988), se obtienen tamaños del efecto medios en las tres subescalas que han sido comentadas anteriormente. Sin embargo, no se obtiene diferencia en el factor $\mathrm{MO}$, lo que puede relacionarse con que el postest se administró al final de la temporada (mes de abril), tras quedar ambos equipos en posiciones medias de la tabla, lo que probablemente afectara a la motivación de los jugadores, pese a la intervención en esta variable mediante el establecimiento de objetivos (López y Moreno, 2018).

En cuanto al factor $\mathrm{CH}$, fue una variable en la que no se intervino, por lo que no se esperaba mejora, sino los mismos resultados en el pretest que en el postest. Sin embargo, se observa menor puntuación en el postest, lo que puede explicarse con que éste se llevo a cabo al final de la temporada, cuando muchos jugadores sabían que iban a cambiar de equipo por promocionar o cambiar de categoría, por lo que es normal que la cohesión disminuya.

Los resultados de este trabajo están en la línea de lo encontrado en la literatura científica en estos últimos años, resaltando la gran importancia que tiene la intervención psicológica en el deporte, proporcionando un mayor bienestar psicológico y un incremento del rendimiento deportivo
(Breslin, Shannon, Haughey, Donnelly y Leavey, 2017; Brown y Fletcher, 2017; Golby y Wood, 2016; Gross et al., 2018). Además, también está en la línea de aquellos estudios que han mejorado, a través del entrenamiento psicológico, variables psicológicas como la motivación, la concentración, la autoconfianza o el nivel de activación (Beckmann and Elbe, 2015; Olmedilla and Domínguez-Igual, 2016), o los recursos y la adquisición de habilidades psicológicas (técnicas, estrategias, etc.) para gestionar mejor su práctica deportiva (McCormick et al., 2018; Olmedilla, Moreno-Fernández et al., 2019; Simonsmeier y Buecker, 2017).

Como principales limitaciones del estudio, se pueden señalar: el número de participantes, el nivel de compromiso de los jugadores, el papel de los entrenadores y el momento de la temporada. Respecto al número de participantes, sería importante poder incrementar el número de deportistas en estudios posteriores. Respecto al nivel de compromiso, la edad y desconocimiento del trabajo psicológico podrían jugar en contra, por lo que sería importante una mayor continuidad en este tipo de intervenciones. En relación al papel de los entrenadores, en este trabajo no se ha intervenido con ellos y habría sido interesante haberlos implicado en el desarrollo del programa o hacerlo en intervenciones posteriores, ya que podría favorecer un mejor clima de grupo y una mayor implicación de los jugadores (Cruz, Mora, Sousa y Alcaraz, 2016; García-Naveira, 2017; Marques, Nonohay, Koller, Gauer y Cruz, 2015; Soriano, Ramis, Cruz y Sousa, 2014). En relación al momento de la temporada en que se realiza el programa de intervención, incidir que resulta muy importante por el peso propio de la competición (clasificación en la liga, resultados, objetivos, etc.), por lo que sería recomendable implementar el programa desde el inicio de la temporada y a lo largo de la misma.

\section{Aplicaciones prácticas}

El presente estudio aporta evidencia sobre la eficacia de las intervenciones psicológicas en el ámbito del deporte base, tal y como muestran los resultados previamente discutidos. Variables como la influencia en la evaluación del rendimiento, habilidad mental o control de estrés, relacionadas con el rendimiento deportivo, pueden verse beneficiadas de este tipo de intervenciones. En este sentido, la figura del psicólogo del deporte se presenta como muy relevante en el buen desarrollo y óptimo funcionamiento de equipos deportivos, contextualizado además en el deporte base, etapa de adquisición de buenas prácticas y hábitos de cara a una futura carrera profesional como deportistas o, simplemente, una sana relación vital con el deporte. 


\section{Referencias}

Abenza, L. González, J., Reyes, L., Reyes, F., Blas, A. y Olmedilla, A. (2014). Descripción y evaluación del entrenamiento psicológico de una deportista de regata clase laser radial. Revista Iberoamericana de Psicología del Ejercicio y el Deporte, 9, 67-92.

Aoyagi, M. W., Poczwardowski, A., Statler, T., Shapiro, J. L. y Cohen, A. B. (2017). The performance interview guide: recommendations for initial consultations in sport and performance psychology. Professional Psychology: Research and Practice 48, 352-360. https://doi.org/10.1037/pro0000121

Beckmann, J. y Elbe, A. M. (2015). Sport psychological interventions in competitive sports. Cambridge Scholars Publishing.

Breslin, G., Shannon, S., Haughey, T., Donnelly, P. y Leavey, G. (2017). A systematic review of interventions to increase awareness of mental health and well-being in athletes, coaches and officials. Systematic reviews, 6(1), 177. https://doi. org/10.1186/s13643-017-0568-6

Brown, D. J. y Fletcher, D. (2017). Effects of psychological and psychosocial interventions on sport performance: a meta-analysis. Sports Medicine, 47, 77-99. https://doi.org/10.1007/ s40279-016-0552-7

Cantón, E. (2016). La especialidad profesional en Psicología del Deporte. Revista de Psicología Aplicada al Deporte y al Ejercicio Físico, 1, e2, 1-12. https://doi.org/10.5093/rpadef2016a2

Carmona, D., Guzmán, J. F. y Olmedilla, A. (2015). Efectos de un programa de formulación de objetivos y moldeamiento del pase en jóvenes jugadores de fútbol. Revista de Psicología del Deporte, 24(1), 81-88.

Clancy, R. B., Herring, M. P., Maclntyre, T. E. y Campbell, M. J. (2016) A review of competitive sport motivation research. Psychology of Sport and Exercise, 27, 232-242. http://dx.doi.org/10.1016/j.psychsport.2016.09.003

Cohen, J. (1988). Statistical power analysis for the behavioral sciences (2nd ed.). Hillsdale, NJ: Erlbaum.

Craig, M. C. (2011). New approaches to studying decision making in sport. Revista de Psicología del Deporte, 20, 689-708.

Cruz, J., Mora, A., Sousa, C. y Alcaraz, S. (2016). Effects of an individualized program on coaches' observed and perceived behavior. Revista de Psicología del Deporte, 25, 137-144.

Díaz, M. I., Villalobos, A. y Ruíz, M. A. (2012). La desensibilización sistemática y técnicas de relajación. En M. Díaz, M. A. Ruíz y A. Villalobos (Eds). Manual de técnicas de intervención cognitivo-conductuales (pp. 237-276). Madrid, España: Desclée de Brouwer.

Eraña, I. (2004). Entrenamiento psicológico con jóvenes tenistas. Revista de Psicología del Deporte, 13, 263-271.

Escolano, E., Herrero, M. L. y Echeverría, R. (2014). La resolución como estrategia de enseñanza para el aprendizaje de la táctica ofensiva en la categoría pre-benjamín de fútbol 8. Revista de Psicología del Deporte, 23, 209-217.

García-Naveira, A. (2017). Entrenamiento psicológico para la mejora del autocontrol en un entrenador de fútbol. Acción Psicológica, 14(1), 27-42. https://doi.org/10.5944/ap.14.1.19252

Gimeno, F., Buceta, J. M. y Pérez-Llantada, M. C. (2001). El cuestionario "Características Psicológicas relacionadas con el Rendimiento Deportivo" (CPRD): Características psicométricas.
Análise Psicológica, 1(19), 93-113. https://doi.org/10.14417/ ap.346

Godoy-Izquierdo, D., Vélez, M. y Pradas, F. (2009). Nivel de dominio de las habilidades psicológicas en jóvenes jugadores de tenis de mesa, bádminton y fútbol. Revista de Psicología del Deporte, 18(1), 7-22.

Golby, J. y Wood, P. (2016). The effects of psychological skills training on mental toughness and psychological well-being of student-athletes. Psychology, 7, 901. http://dx.doi. org/10.4236/psych.2016.76092

Gómez-Espejo, V., Aroca, B., Robles-Palazón, F. J. y Olmedilla, A. (2017). Formación integral en la cantera del Real Murcia C. F.: Tutorías psicológicas. Revista de Psicología Aplicada al Deporte y al Ejercicio Físico, 2, e11 1-11. https://doi.org/10.5093/ rpadef2017a11

González, A. M. (2006). El uso de la visualización en el deporte. En E. J. Garcés de los Fayos, A. Olmedilla y P. Jara (Eds). Psicología y Deporte (pp. 279-295). Murcia, España: Diego Marín.

González, J. y Garcés de los Fayos, E. (2009). Plan de entrenamiento psicológico en el deporte de la petanca: en búsqueda del rendimiento grupal óptimo. Revista de Psicología del Deporte, 18(1), 87-104.

Gross, M., Moore, Z. E., Gardner, F. L., Wolanin, A. T., Pess, R. y Marks, D. R. (2018). An empirical examination comparing the mindfulness-acceptance-commitment approach and psychological skills training for the mental health and sport performance of female student athletes. International Journal of Sport and Exercise Psychology, 16, 431-451. https:// doi.org/10 $.1080 / 1612197 \times .2016 .1250802$

Larkin, P., Mesagno, C., Berry, J., Spittle, M. y Harvey, J. (2018). Video-based training to improve perceptual-cognitive decision-making performance of australian football umpires. Journal of Sports Science, 36, 239-246. https://doi.org/70.108 0/02640414.2017.1298827

Lindern, D. (2016). Desenvolvimento de uma intervenção com foco preventivo baseada na terapia cognitivo-comportamental e na psicologia positiva para atletas de futebol adolescentes. (Tesis Dissertação de Mestrado). Portugal: Pontícia Universidade Católica do Rio Grande do Sul.

López, J. C. y Lozano, F. J. (2006). Activación, estrés y ansiedad. En E. J. Garcés de los Fayos, A. Olmedilla y P. Jara (Eds). Psicología y Deporte (pp. 191-204). Murcia, España: Diego Marín.

López, J. M. y Moreno, R. (2018). Tipos de motivación y su relación con las expectativas de compaginar deporte y estudios en jóvenes jugadores de fútbol. Revista Española de Educación Física y Deportes 421, 59-70.

Lorenzo, J., Gómez, M. A., Pujals, C. y Lorenzo, A. (2012). Análisis de los efectos de un programa de intervención psicológica en jóvenes jugadores de baloncesto. Revista de Psicología del Deporte, 21, 43-48.

Marques, M., Nonohay, R., Koller, S., Gauer, G. y Cruz, J. (2015). El estilo de comunicación del entrenador y la percepción del clima motivacional generado por los entrenadores y compañeros. Cuadernos de Psicología del Deporte, 15(2), 47-54. https:// doi.org/10.4321/S1578-84232015000200006

McArdle, S. y Moore, P. (2012). Applying evidence-based principles from CBT to sport psychology. The Sport Psychologist, 26, 299-310. https://doi.org/10.1123/tsp.26.2.299 
McCormick, A., Meijen, C., Anstiss, P. A. y Jones, H. S. (2017). Self-regulation in endurance sports: theory, research and practice. International Review of Sport and Exercise Psychology. https://doi.org/10.1080/1750984X.2018.1469161

McCromick, A., Meijen, C. y Marcora, S. (2018). Effects of a motivational self-talk intervention for endurance athletes completing an ultramarathon. Sport Psychologist, 32, 42-50. https:// doi.org/10.1123/tsp.2017-0018

Mora, J. A., Zarco, J. A. y Blanca, M. J. (2001). Atención-concentración como entrenamiento para la mejora del rendimiento deportivo en jugadores profesionales de fútbol. Revista de Psicología del Deporte, 10(1), 49-65.

Morilla, M. (2006). Entrenamiento de la atención y concentración en el deporte. En E. J. Garcés de los Fayos, A. Olmedilla y P. Jara (Eds.), Psicología y Deporte (pp. 315-333). Murcia, España: Diego Marín.

Mostaan, M., Sanatkaran, A. y Bahari, S. M. (2015). The Effects of Behavioral Intervention on Mood in Disabled Professional Basketball Players. Journal of Novel Applied Sciences, 4, 797-803.

Navarrón, E., Godoy-Izquierdo, D., Vélez, M., Ramírez-Molina, M. J. y Jiménez-Torres, M. G. (2017). Implementación de una intervención psicológica en fútbol base, satisfacción subjetiva de los deportistas y experiencias de pasión, competencia percibida y compromiso deportivo en relación con la intención de práctica futura. Revista Iberoamericana de Psicología del Ejercicio y el Deporte, 12(1), 59-69.

Nieto, G. y Olmedilla, A. (2001). Planificación del entrenamiento psicológico en atletas de élite: un caso en marcha atlética. Revista de Psicología del Deporte, 10(1), 127-142.

Olmedilla, A., Bazaco, M. J., Ortega, E. y Boladeras, A. (2011). Formación psicológica en futbolistas jóvenes. Relación con el bienestar psicológico percibido, la ansiedad y la satisfacción. Revista Científica Electrónica de Psicología, 12, 221-237.

Olmedilla, A. y Domínguez-Igual, J. J. (2016). Entrenamiento psicológico para la mejora de la atención y la autoconfianza en un futbolista. Revista de Psicología Aplicada al Deporte y al Ejercicio Físico, 1, e1. https://doi.org/10.5093/rpadef2016a4
Olmedilla, A., Moreno-Fernández, I. M., Gómez-Espejo, V., Robles-Palazón, F. J., Verdú, I. y Ortega, E. (2019). Psychological intervention program to control stress in young soccer players. Frontiers in Psychology, 10, 2260. https://doi.org/10.3389/ fpsyg. 2019.02260

Olmedilla, A., Ortega, E., Andreu, M. D. y Ortín, F. J. (2010). Programa de intervención psicológica en futbolistas: evaluación de habilidades psicológicas mediante el CPRD. Revista de Psicología del Deporte, 19, 249-262.

Olmedilla, A., Ortega, E., Boladeras, A., González, J. y Serpa, S. (2015). Psychological Training in Sailing: Performance Improvement for the Olympic Classification Phase. Universal Journal of Psychology, 3(4), 122-131. https://doi.org/10.13189/ ujp. 2015.030404

Olmedilla, A., Ruiz-Barquín, R., Ponseti, F. J., Robles-Palazón, F. J. y García-Mas, A. (2019). Competitive Psychological Disposition and Perception of Performance in Young Female Soccer Players. Frontiers in Psychology, 10, 1168. https://doi. org/10.3389/fpsyg.2019.01768

Olmedilla, A., Torres-Luque, G., García-Mas, A., Rubio, V. J., Ducoing, E. y Ortega, E. (2018). Psycological profiling of triathlon and road cycling athletes. Frontiers of Psychology. 9, 825. https://doi.org/10.3389/fpsyg.2018.00825

Simonsmeier, B. A. y Buecker, S. (2017). Interrelations of imagery use, imagery ability, and performance in young athletes. Journal of Applied Sport Psychology, 29(1), 32-43. http://dx.doi.org/ 10.1080/10413200.2016.1187686

Soriano, G., Ramis, Y., Cruz, J. y Sousa, C. (2014). Un programa de intervención individualizado con entrenadores de fútbol. Cuadernos de Psicología del Deporte, 14(4), 99-106. https:// doi. org/10.4321/S1578-84232014000300011

Torregrosa, M. y Cruz, J. (2006). El deporte infantil como base de la carrera deportiva de adultos activos y deportistas de élite. En E. Garcés de los Fayos, A. Olmedilla y P. Jara (Eds.), Psicología y Deporte (pp. 585-602). Murcia, España: Diego Marín. 